\title{
Internet-Based Support and Coaching for Adolescents and Young Adults with Neuropsychiatric Disorders-The Implementation of an Intervention from an Organizational Perspective
}

\author{
Nanna Gillberg, Elisabet Wentz \\ Gillberg Neuropsychiatry Centre, Institute of Neuroscience and Physiology, University of Gothenburg, \\ Gothenburg, Sweden \\ Email: nanna.gillberg@gu.se
}

How to cite this paper: Gillberg, N. and Wentz, E. (2017) Internet-Based Support and Coaching for Adolescents and Young Adults with Neuropsychiatric DisordersThe Implementation of an Intervention from an Organizational Perspective. Health, 9, 6987.

http://dx.doi.org/10.4236/health.2017.91006

Received: November 29, 2016

Accepted: January 10, 2017

Published: January 13, 2017

Copyright (c) 2017 by authors and Scientific Research Publishing Inc. This work is licensed under the Creative Commons Attribution International License (CC BY 4.0).

http://creativecommons.org/licenses/by/4.0/

\begin{abstract}
There is a documented and often unmet need for interventions aimed at supporting young people with attention-deficit/hyperactivity disorder (ADHD) and/or autism spectrum disorder (ASD) in their transition between adolescence and adulthood. Difficulties with social interaction, initiation difficulties, and impairments in executive function can complicate visits at a clinic, i.e. traditional treatment, for individuals with neuropsychiatric disorders (NPD). A model for internet-based support and coaching aimed at young people with ASD and/or ADHD was developed and tested at three treatment sites in western Sweden. The implementation was analyzed against an inventory according to which implementations are more likely to be successful if an intervention: 1) has advantages compared to other existing methods, 2) matches the individual and organizational values, norms and work practices of its recipients, 3 ) is perceived as easy to use, and 4) is adaptable to local conditions and the recipients' needs. Data were collected through group interviews with professionals involved in the implementation of the intervention. The implementation of the intervention showed promising results on measures such as access, delivery/quality of healthcare services, and equality of distribution of healthcare services. The identified impediments to successful implementation related to a wide range of factors and levels, including the design of the intervention, technical issues, attitudes of staff, organizational culture, and organizational structure at the implementation sites in terms of patient stock, work division, and resource allocation. The results are consistent with previous studies that stress the need for multi-component implementation strategies.
\end{abstract}




\section{Keywords}

ASD, ADHD, Internet-Based Support, Implementation, Intervention

\section{Introduction}

This study presents a follow-up of the implementation of an intervention combining the technique of coaching with digital technology_individual online coaching and support. In a pilot study, the intervention was offered to adolescents and young adults with attention-deficit/hyperactivity disorder (ADHD) and/or autism spectrum disorder (ASD) at three physical treatment sites in western Sweden.

There is a documented and often unmet need for interventions aimed at supporting young people with ADHD and/or ASD in their transition between adolescence and adulthood [1] [2] [3] [4]. The transition from adolescence to adulthood is a critical stage in any individual's life. For those with ADHD and/or ASD it can be particularly complicated. Increased expectations on the individual to be independent coincide with the loss of structure provided to daily life by school. Levine (2005) has highlighted the tendency of young adults with ADHD to remain in their teens for an extended period of time [5]. Several studies have also shown that adolescents with ASD suffer from high levels of anxiety [6] [7]. In spite of documented difficulties, many individuals with ADHD and/or ASD lack access to support from people with special knowledge about neuropsychiatric disorders (NPD). This group often falls through the gap due to not meeting referral criteria for mental health services [8]. Consequently they do not receive any support as young adults.

Insufficiencies in the support and interventions to individuals with NPD may also relate to the degree to which existing treatments are adapted to the needs of this patient group. Difficulties with social interaction, initiation difficulties, and impairments in executive function are commonly shared characteristics that can complicate visits at a clinic, i.e. traditional treatment, for individuals with NPD. Particularly face-to-face communication may be problematic in individuals with ASD [9].

Previous research has stressed the importance of a multimodal treatment approach for reaching individuals with NPD [10]. Internet presents an opportunity to design support measures to patient groups who tend to find face-to-face meetings in a clinic stressful [11]. Coaching is a method suggested in NPD to facilitate the transition from adolescence into adult life [12], and one that has been recommended to improve social skills in adolescents and young adults with ASD [13]. By eliminating non-verbal cues such as body language tone of voiceintegral parts of face-to-face encounters-internet-based support allows patients to focus on reciprocal communication while at the same time lowering their stress level. Being able to conduct the interaction from a familiar environment may al- 
so help to reduce the patient's anxiety level [11].

Against this background a model for internet-based support and coaching aimed at young people with ASD and/or ADHD was developed and tested within the scope of a pilot study. The implementation of this intervention is the study object of the current article. The gap between research findings and practice is among the most consistent findings in studies on health services [14] [15], indicating a serious need for research evaluating transitional care practices aimed at young people with complex healthcare needs [16].

\section{Pilot Study-Implementation of a Model for Internet-Based Support and Coaching at Three Treatment Sites in Western Sweden}

Seeking to identify and introduce a user-friendly and convenient form of support for young individuals with NPD, a model for internet-based support and coaching was developed using previous knowledge and input from the target group in the development. The pilot study was designed with the aim of 1) developing a model for internet-based support and coaching for adolescents and young adults with NPD and 2) validating the model. The study was initially carried out at a treatment site in western Sweden. Current or previous patients at the treatment site were offered the intervention. Twelve individuals of $15-26$ years took part in the trial (two of whom dropped out before the completion of the intervention), five girls/women and seven boys/men (four girls and six boys completed the project). To be included the individuals had to fulfill a diagnosis of ADHD and/or ASD. Six of the patients had ASD with or without ADHD; ten patients had ADHD with or without autism. The trial included a total of 14 chat sessions and two visits at a clinic. The coaches were either clinical psychologists or educational therapists. Exclusion criteria comprised intellectual disability, current psychosis, current alcohol and/or substance abuse/dependence, severe dyslexia and/or severe psychosocial problems (e.g. criminality). While major depression was among the exclusion criteria, exceptions were made if an individual was highly motivated to take part in the intervention and there was no indication of his/her state deteriorating. Before the individual started with the internet-based support and coaching, a compulsory face-to-face meeting with the assigned coach at the treatment site was conducted. At this meeting the coach and the prospective participant discussed the specific issues they would be working on during the course of the intervention based on the individual's requirements. Finding employment and gaining an income, getting started, organizing and structuring one's daily life, understanding oneself through the disorder, and grasping and managing social relations were among the identified themes. In addition to the online chat sessions the participants had two face-to-face meetings with their coach (approximately three and six weeks into the intervention). Upon completion of the eight weeks, participants were interviewed with regard to the quality of the intervention (the Patient perspective of Care and Rehabilitation process POCR). Participants also filled out self-report questionnaires before 
and after the intervention and six months later [Sense of Coherence (SOC-13), Rosenberg Self Esteem Scale, Manchester Short Assessment of Quality of Life (MANSA), Montgomery Åsberg Depression Rating Scale (MADRS) and the Hospital Anxiety and Depression Scale $(H A D)$ ). The evaluation showed improvement of the participants' self-esteem, sense of coherence, and subjective quality of life, indicating that internet-based support and coaching can be an important complement to other interventions for young people with ASD and/ or ADHD [10].

Supported by the encouraging results of the first unit's trial, the pilot study was extended to include two additional sites in western Sweden. At this stage in the implementation process, the maximum age for participants was raised to 30 years. The coaches at the two additional sites were clinical psychologists, occupational therapists, social workers, and educational therapists. In all other respects the design of the intervention and the follow-up remained the same as in the initial stage of the trial. The study was approved by the Regional Ethical Review Board at the University of Gothenburg, Sweden (reference number 013-08, plus two supplementary amendments that approved the extension of the trial to two additional treatment sites).

\section{Methodology}

\subsection{Data Collection}

To gather information on the intervention and its implementation from an organizational perspective, three semi-structured group interviews were conducted with professionals involved in the implementation of internet-based support and coaching at the three treatment sites included in the trial. A total of seven individuals were interviewed (two interviews with two interviewees and one interview with three interviewees) - three coordinators, three coaches and the project manager. In her capacity of the studied trial's project manager, the second author of the article was included among the interviewees. To address the potential problem of her dual roles and avoid the risk of interference or bias, the interview in which the second author took part was used solely to provide background and an overview of the project as a whole.

The choice of focus group interviews was motivated by the heuristic value of interaction between the members of a certain social group (in this case healthcare workers at the studied treatment sites) offered by the technique. Group interviews may allow for the expression of inter-subjective representations that reflect the images and beliefs of a particular social group [17]. As such they lend themselves well to exploring the article's research questions. The interviewee composition was designed to ensure that information pertaining to both the intervention and the implementation process could be gathered. The interviews were conducted using semi-structured questions that helped to map out the areas relevant to the study while simultaneously allowing the interviewer and interviewees room to diverge in order to develop specific issues and lines of thought [18]. 


\subsection{Data Processing}

The empirical material was analyzed using thematic analysis. Thematic analysis involves searching for common patterns or themes in an empirical material, in a process that involves the following steps: getting familiarized with data, identifying initial codes, searching for themes, reviewing themes, defining and naming themes, and producing a report. Rather than having to be founded in quantifiable measures, a theme is defined mainly through its ability to capture something important in relation to the overall research question [19]. For the current article the themes were identified using both frequency and relevance to the main research question as selection criteria.

\subsection{Purpose, Research Questions and Scope}

A trial can be successful or fail depending on elements pertaining to the intervention, the implementation, or a combination of both. When evaluating the implementation of new methods or practices a distinction must therefore be made between the intervention at hand and the implementation process [20] [21] [22]. In the present context intervention refers to the internet-based support and coaching as a method. Implementation, meanwhile, is defined as a specified set of activities designed to put into practice an activity or program of known dimensions [20]. Although the primary focus is on the implementation process, the intervention itself is addressed as the implementation and its barriers and facilitators are seen in relation to the intervention and its characteristics.

Research questions: How is the implementation of internet-based support and coaching perceived by the professionals involved in implementing it?

Data collection: 3 treatment sites

Data analysis:

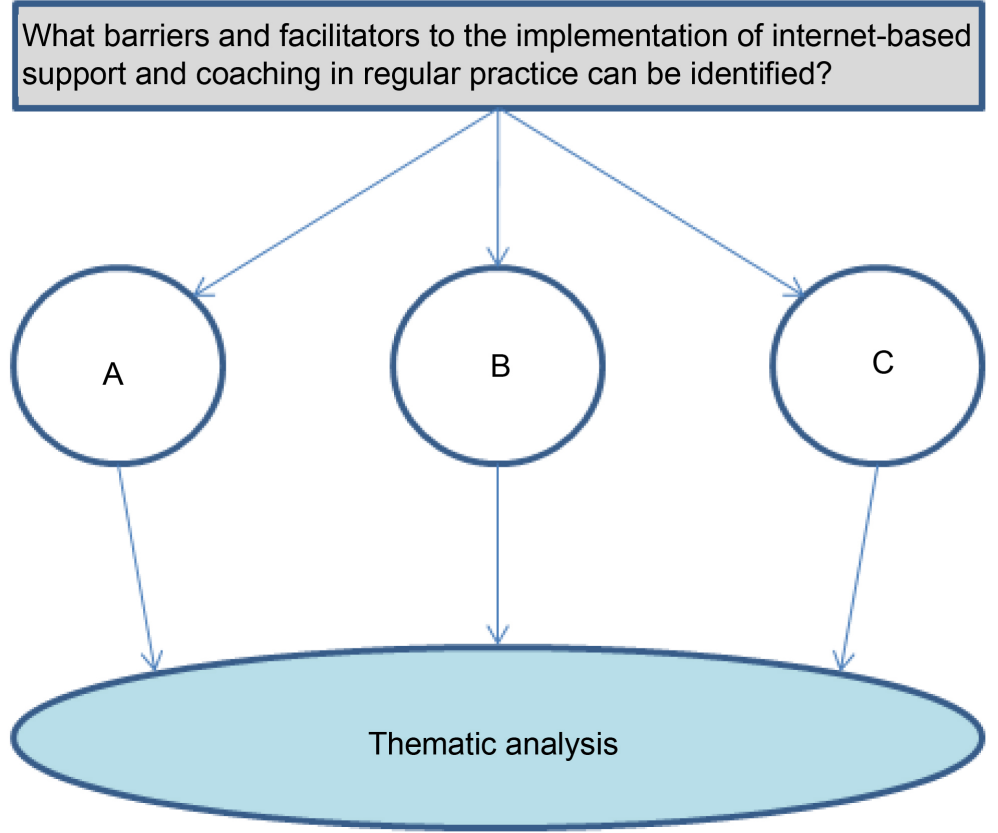

Figure 1. Research questions, data collection and analysis. The letters A, B and C correspond to the three treatment sites. 
The following research questions provide the starting point of the study:

How is the implementation of internet-based support and coaching perceived by the professionals involved in implementing it?

What barriers and facilitators to the implementation of internet-based support and coaching in regular practice can be identified? The research questions, data collection and data analysis are shown in Figure 1.

\section{E-Health and Telemedicine}

Over the last few decades staggering healthcare costs have been a recurring theme in public policy discussions. The issue of how to lower costs and make delivery of healthcare services more efficient hence has become a primary concern. Following the transition from analogue to digital technology and the consequent decrease in the cost of various forms of Information and Communication Technology (ICT), telemedicine has sparked a wide interest among policy makers and health officials [23]. While widely used there is no clear consensus on the meaning of the concept of telemedicine. The authors of a literature review identified 104 peer-reviewed definitions of the term [24]. In the current article the simple and straightforward definition proposed by Lehoux et al. (2002) identifying telemedicine as "the use of information technology to support delivery of healthcare from a distance" will be employed [25]. The application of ICT in healthcare (including telemedicine, electronic medical records, mobile health, and elearning tools) is commonly referred to as e-health [26]. As with telemedicine this is a widely used concept with no clear and agreed upon definition and meaning.Oh et al. (2005) identified 51 unique definitions covering a set of distinct concepts, including health, technology and commerce [27]. The definition most frequently quoted, and the one employed in the current article, was proposed by Eysenbach, and classifies e-health as:

"an emerging field in the intersection of medical informatics, public health and business, referring to health services and information delivered or enhanced through the Internet and related technologies. In a broader sense, the term characterizes not only a technical development, but also a stateof-mind, a way of thinking, an attitude, and a commitment for networked, global thinking, to improve healthcare locally, regionally, and worldwide by using information and communication technology". (Eysenbach, 2001, e20)

The use of information technologies in the delivery of healthcare has been described as essential for high quality and cost-effective healthcare services. Telemedicine has been said to have the potential to reduce costs and empower patients while improving healthcare services in a number of ways including enhanced access, quality and efficiency [28] [29] [30] and improved ability to meet the needs of underserved populations in both industrialized and developing countries [31] [32] [33] [34]. In spite of the talk of the advantages of e-health interventions however, the implementation of such interventions has often proved problematic and failing to deliver the forecasted benefits [27]. To realize the po- 
tential of e-health initiatives and make them permanent parts of the healthcare services on offer, further research on e-health implementations is needed. A literature review on barriers and facilitators in e-health implementations [35] identified a number of key barriers to successful implementation of e-health initiatives, including inadequate information management and inter-agency cooperation, intrusive technology/rigidity of systems, costs and lack of testing of systems, issues connected with health professional interaction and organizational factors. Among the facilitators, inter-agency co-operation, flexibility, ease of use and organizational willingness were noted.

The vast majority of existing research on telemedicine has focused on clinical, technical, and economic aspects. Social and organizational issues, meanwhile, have been sparsely addressed [36]. To address technology in this way implies a focus on how local conditions of use partakes in and contributes to organizational and economic conditions. It also includes looking at whether telemedicine is aligned with or deviates from the existing professional and institutional organization of healthcare [37] [38].

\section{Interventions and Implementation}

Implementation processes are complex and generally involve simultaneous consideration to a wide range of human, financial, and technical variables [39]. While implementation and intervention comprise two distinct entities they are closely linked to one another in that the intervention impacts the likelihood of an implementation being successful. Implementation success can be defined as employee commitment to and consistent use of new, better practices [40]. Based on an inventory of previous research Guldbrandsson (2007) presents a list of factors shared by successfully implemented interventions. According to this inventory successful implementations are more likely to occur if an intervention has relative and visible advantages compared to other existing methods [21] [41] [42], if it matches the individual and organizational values, norms and work practices of its recipients [21] [22] [41] [42] [43], if it is perceived as easy to use [21] [22] [41] [44], and if it is adaptable to local conditions and the recipients' needs [21] [41] [45].

\section{Results}

Under this heading the presentation of the empirical material is sorted using some sub-headings. These reflect the themes derived through the interviewees' narratives.

\subsection{Mediated Communication as a Qualitative and Quantitative Advantage}

According to staff at the treatment sites, the internet-based support and coaching, through its mediated interaction and by enabling support to be received from the comfort of one's own home, offers a delivery of health services that is well suited to the specific needs associated with NPD such as ASD and ADHD. 
Several of the interviewees offered descriptions of the ways in which traditional visits at clinics can be strenuous for this group of patients. Getting to a clinic, sitting in the waiting room with other people, and then be in a face-to-face situation with the expectations of sociability (interpreting non-verbal social cues etc.), are elements that can all be perceived as difficult and hence impede treatment. When describing the mediated internet-based interaction in relation to traditional face-to-face interaction one coach concluded that "it doesn't steal nearly as much energy". Stripping communication of its non-verbal component interviewees said has a positive effect on the patient's ability to benefit from the intervention. Energy that in face-to-face meetings would be spent trying to interpret and conform to social norms and expectations can instead be spent on the communication.

Several interviewees challenged the assumption often made from a neurotypical perspective, that the quality of communication is higher in face-to-face interaction than in mediated interaction. Rather than having a negative impact on the quality of the communication, the mediation element was said to increase the quality of the communication. One coach reflected on this in the following way: "While I might feel that you lose a dimension when you strip off the elements of eye contact and interpreting the other person, the patient might feel the opposite." Several coaches mentioned the clarity of written communication as a feature particularly well suited to the needs of many patients within the autism spectrum. One interviewee stated that: "Things get clearer and more to the point when writing". Several interviewees also maintained that patients tended to share more and give richer descriptions in the internet-based chat than in faceto-face conversations. One interviewee explained this by stating that "it is easier to talk about difficult things when in the safety of your own home". The difference between face-to-face meetings and the online chat sessions was described as follows by an interviewee at another treatment site: "A bit more dry and formal in face-to-face conversations, but in the chat session really enjoyable and fun."

Internet-based support and coaching potentially marking the difference between receiving and not receiving an intervention is a theme brought up by some interviewees. In many districts the uptake area of healthcare services covers a vast geographical region, which makes extensive traveling on the part of the patient a prerequisite for traditional treatment at a clinic. This further complicates traditional interventions. According to one interviewee the mediated nature of the communication in online coaching was a condition for some of the sessions taking place at all: "Patients have said that they would probably have cancelled several times if the appointments had been taking place face-to-face at a clinic."

\subsection{Technical Issues and the Issue of Technology}

Interviewees at the three treatment sites reported that technical issues had presented a persistent problem throughout the trial. Incompatibility issues stemming from patients having newer computer systems and more efficient browsers 
at home than the older systems carried by the treatment sites were said to have constituted one such issue in the implementation of online chat support. Several interviewees reported that the technical glitches had affected both the quality of the chat support and the motivation among participants, for instance by slowing down the conversations and creating uncertainty as to whether the chat partner had received and read your responses. "You didn't know whether the absence of response was due to technical issues." Not being able to rely on the technology to work properly had also been a cause for stress and extra work for the trial's professionals, who expressed that they felt a need to turn up early for the chat sessions to ensure that everything would be working when the participant joined the chat session.

Some interviewees described the implementation of the intervention as more time-consuming than they had originally anticipated, e.g. by requiring considerable time for administrative tasks-a notion that was linked in part to the continual technical difficulties. The mere implementation of new technology in a previously low-tech profession was suggested as another potential reason behind the perception of the intervention as time-consuming.

\subsection{The Intervention Related to Societal and Organizational Norms and Work Practices}

"It's more on equal terms", is a statement made by two interviewees in reference to the intervention. They credited the method with balancing out an unequal balance of power in the patient-caregiver relationship by adapting to the needs and abilities of the patient. One coach described the coaching sessions as a way of "meeting patients on their home turf", referring both to the online environment and to the communication taking place in the participants' home environment.

While complying with expectations of increased equality in the patient-caregiver relationship on a societal level, the intervention was depicted as not fully aligned with local norms and work practices on an organizational level. The interviewees at one treatment site recounted that information about the internet-based support and coaching program had been met by negative reactions by some members of staff. Branding it "too exclusive", these staff members were said to have objected to the intervention on the basis of it allocating considerable resources to very few individuals. While this position was said to have been held mainly by staff that were not working with patients with NPD and that would hence not be directly involved in the trial, it could still have had a bearing on the implementation success as these employees were expected to contribute to the recruitment process by referring eligible patients to the trial.

Interviewees interpreted the negative attitudes held by some of their coworkers in two main ways: Firstly, as a reaction to an emerging interest in neuropsychiatric diagnoses in society as a whole. Members of staff not working with this patient group were suggested to perceive their position threatened by the emphasis on neuropsychiatry by policy makers and the media. Secondly, the negative attitudes were attributed to a certain organizational culture within some 
work units. Difficulty in letting go of patients and an unwillingness to refer "your" patients to someone else were described as expressions of a culture within habilitation services and were said to have had an adverse effect on the implementation of the internet-based support and coaching. One interviewee argued that "the tradition within habilitation services stipulates that you keep your patients over time and keep them to yourself." Several interviewees likened the negative reactions to the intervention to a "collision between old and new", adding that, "the chat support represents a new way of thinking about treatments".

\subsection{Intensity and Duration}

With its two sessions per week the intervention constituted a relatively high-intensity form of support. The majority of the interviewees described the intensity overall as a positive feature, stating that the two weekly sessions allowed for everyday life events to be dealt with and followed up as they happened. "Things do not get out of date and you can get right to the point without having to recapitulate what was said in the previous session", one interviewee stated while contrasting the intervention to traditional forms of intervention, where the patients would typically come in for a therapy session once every three weeks.

While the high intensity was said to have a positive impact on the quality of the intervention, both the intensity and the duration of the intervention were said to have deterred some candidates from participating and had posed problems in terms of following through for others. One interviewee described the intensity as a feature that at first attracted patients to the intervention, but which over time came to be perceived as too much. Previous commitments such as school, work, and job practice were stated as obstacles to participation, indicating that the long-term commitment of having to sign up for eight weeks of quite intense treatment presented a problem in some cases. For some individuals with $\mathrm{ADHD}$, the requisite continuity (to stick with the appointments and complete all the chat sessions) was reported to have presented a problem. On the other hand, and as pointed out by some interviewees, the intervention provided structure and continuity to a group of patients who often struggle in these areas.

\subsection{The Recruitment Process}

The treatment sites actively recruited participants: identified and contacted potential participants and informed them about the project and the conditions for participation. Participants were recruited from the stock of patients at each site, also employing the help of habilitation services and adjacent units that were asked to develop a list of eligible patients. Patients who had been selected as viable candidates were approached and asked if they would be interested in participating in the project. Interviewees at all treatment sites expressed that the interest among the approached patients was lower than they had anticipated. "It felt like we had to chase", stated one interviewee with another interviewee adding that "the response was worse than we had expected." Candidates' reasons for not 
taking part in the intervention trial included incompatibility with previous commitments, not wanting any contact with welfare agents, and apprehension regarding internet security.

A few interviewees raised concerns regarding the usefulness of targeting newly diagnosed patients, arguing that the diagnosis may have to sink in before the individual can be open to offers of treatment that relate to the understanding of one's diagnosis.

Interviewees at all treatment sites stressed the importance of raising awareness about the intervention throughout the organization for achieving a smooth recruitment process. Lack of awareness of the intervention and its benefits was thought to have affected the way some staff members informed patients about it and to have stopped some from informing about the intervention altogether. Interviewees acknowledged problems of getting staff to adapt to the thought of internet-based support and coaching and incorporating it as one treatment in the portfolio of treatments offered, noting that: "It is not in our repertoire of treatments yet." "We have to do a lot of lobbying, talk about this." Frontline gatekeepers and management were put forward as categories of particular importance for the adoption of the new intervention. For the intervention to be successful several interviewees concluded that the frontline gatekeepers had to be aware of the internet-based support and coaching and include it in their thinking as an available intervention when offering support to patients. Some interviewees also stressed the importance of management being familiar with the intervention, as this would ensure that the operative teams were being reminded of the chat support.

\subsection{Matching the Patient Base with the Intervention}

Some interviewees connected the recruitment difficulties to the patient stock available at the treatment sites and questioned whether their unit was suited to the intervention. Suggesting that the inclusion criteria might not be adapted to the treatment sites taking part in the trial, one interviewee proposed that the intervention "would work better in habilitation services". Several interviewees reported having to exclude a number of potential participants due to comorbidity and the need for more extensive support. This was also mentioned in relation to the aim of the pilot study to reach those individuals who are least likely to be reached with traditional interventions. This group includes individuals who find leaving home overpowering and whose societal contacts are therefore very limited. Several interviewees expressed regret that these "hard-core" patients, who are generally not reached by traditional interventions, were largely missing in the internet-based support pilot as well.

\section{Discussion}

Sorting under the umbrella of telemedicine and e-health, the internet-based support and coaching represents a form of distance healthcare [46]. As such it embodies both a method to access health services and a means to deliver such 
services [47].

Analyzed against Guldbrandsson's (2007) inventory of factors associated with successfully implemented interventions the internet-based support and coaching reveals both benefits and barriers.

An intervention is more likely to be successfully implemented if it has relative and visible advantages compared to other existing methods [21] [41] [42].

The intervention showed promising results on several measures commonly named as benefits of telemedicine, such as improved access, enhanced delivery/ quality of healthcare services, and equality of distribution of healthcare services [24]. Compared to traditional treatment at a clinic, internet-based support and coaching may have some both quantitative and qualitative benefits. A quantitative benefit lies in the ability to reach individuals that might otherwise, for a combination of social and geographical reasons, receive no support. The qualitative benefit consists of the internet-based support providing an intervention that, in many ways, is better suited to the abilities, difficulties, and needs of the recipients than traditional interventions. The commonly recognized perception of face-to-face interaction as qualitatively superior to mediated interaction can be said to rest on neurotypical assumptions that are hence contextual and not necessarily applicable in this situation.

An intervention is more likely to be successfully implemented if it is perceived as easy to use [21] [22] [41] [44].

Compared with traditional treatment at a clinic the intervention can be said to constitute a relatively easy-to-use method as it bridges geographical distances and accommodates to social and executive difficulties in the targeted patient group. Detracting from the easy-to-use character however was the enduring technical problems throughout the implementation trial. Problems of incompatible systems and communication glitches and delays affected both the quality of the intervention and the motivation of the participants. Addressing the technical issues will be of vital importance for the successful implementation of the intervention in regular practice.

An intervention is more likely to be successfully implemented if it matches the individual and organizational values, norms and work practices of its recipients [21] [22] [41] [42] [43].

The interviews highlight the need for e-health initiatives to take into account prevailing notions of what constitutes high-quality care and service delivery, on a societal as well as on an organizational level. The Swedish healthcare system is regulated by the Health and Medical Services Act (SFS 1982: 763), in which is stated that healthcare should be accessible, built on respect for the patients' autonomy and integrity, and facilitate positive patient-healthcare provider contacts. The interviewees depict internet-based support and coaching as an intervention that largely fulfill these criteria. The professionals responsible for implementing the intervention hence perceive it as aligned with a currently prevailing, research-supported [48] [49] and politically advocated, egalitarian and participatory approach to healthcare, for example articulated in the patient- 
centered care concept [50] [51] [52] [53]. While described as in cultural concordance with its recipient context on a societal level, the intervention on an organizational level seems to partly deviate from norms, values, culture, and work practices at the studied treatment sites. Here the recruitment process calls for particular attention. Interviewees relate the experienced difficulties in recruiting participants to the trial partly to organizational culture and to perceived changes in power balance and resource allocation between professional groups within the organization. This interview theme supports previous research calling for studies on the effects of implementing e-health on healthcare providers' interactions, roles and responsibilities [54] as well as research stressing the importance of new interventions corresponding to the values and skills of their users [55]. Implementations of new methods generally bring about changes in resource allocations and power balance. Hence change is not a neutral act but one that carries a political element. It follows that resistance to change often accompanies efforts to implement new practices [56] [57] [58] [59]. Employees' attitudes towards a change event are one of the most critical factors to successful implementations [60]. The reported resistance to the intervention among some staff indicates a need for further work on attitudes and raising awareness in order to achieve staff commitment to the intervention throughout the organization. Achieving a smooth recruitment process is closely linked to whether members of the implementing organization are aware of and not opposed to the intervention. For the chat support to have an impact it must be integrated in the daily operations of the treatment sites, not only on a structural level but also in the minds of the organizational members as part of the various treatments available to patients. Gatekeepers and management are brought forward in the interviews as two groups of particular importance for successful implementation.

Recruitment difficulties can further be related to the patient stock at the implementation sites. From a patient stock perspective it is suggested that habilitation services should be the preferred environment in which to implement the intervention. At the same time some habilitation services are reported to have shown ambivalence towards the intervention based, in part, on its focus on and resources allocated to patients with NPD.

Future recruitment processes and inclusion criteria will require adaptation to the patient stock and to the emerging notion that overlap of symptoms and co-existence of diagnoses in patients with NPD constitute the rule rather than the exception [61] [62]. Matching the intervention with participants is likely to constitute a key task in an extended implementation.

The interviewees' statements are consistent with the results of previous research in stressing the need for multi-component implementation strategies [63] [64] [65] [66]. Durable change generally requires a comprehensive plan, targeting different barriers to change, and strategies aimed at different levels (professional, team, patient, organization, and wider environment) [67]. In the present context this is demonstrated by the impediments to successful implementation pertaining to a wide range of factors and levels, such as the design of the inter- 
vention, technical issues, attitudes of staff, organizational culture, and organizational structure at the implementation sites in terms of patient stock, work division, and resource allocation.

\section{Limitations}

This article approaches the implementation of an intervention from an organizational perspective, thus placing the focus of the study on the experiences of the organizational members involved in the trial. Although it is of essence to obtain and convey the recipients'/patients' perspective, it falls outside the scope of this article due to its organizational theory focus. The patient-side is however accounted for through the interviews and patients' written evaluations of the quality of the intervention, as these in part comprise the basis for the interviewees' statements. More importantly, the patients' perspective has been the focus of several previous works produced within the frame of this research project [10] [68] [69].

The studied implementation taking place with in a research study setting presents a limitation to the current study. A common criticism of treatment research deals with the discrepancy between clinical practice and controlled research trials. As key conditions and characteristics of treatment research depart noticeably from those in clinical practice it has been questioned whether the results can be generalized to regular practice [70]. Several of the current study's implications call for comments as they may be associated with the research project status and not necessarily applicable had the intervention instead taken place within regular practice. One such implication is the limited scope for flexibility allowed as a result of the strict guidelines of the research project. In her inventory of factors associated with successfully implemented interventions Guldbrandsson (2007) concludes that methods that can be adapted to local conditions and recipients' needs are easier to implement than more rigid ones [71]. The research structure required coordinators to, within a given timeframe, secure a certain number of participants. This might have affected the selection process by creating a propensity to cling to potential participants while overlooking signs of non-commitment to the trial. As difficulty to attract committed participants characterized the recruitment process, the conditions of the trial could hence have contributed to a somewhat adverse selection of participants. While interviewees emphasized the principle of letting patients' needs comprise the starting point for any intervention, this principle might to some extent have been compromised by the implementation's presence within a research project frame.

Interviewee statements of having to eliminate potential recipients from the intervention due to comorbidity are aligned with results of treatment research noting that patients in controlled trials tend to have less severe disorders and fewer comorbid disorders than patients who routinely come to treatment [72]. This brings into question whether the intervention was properly accounting for comorbidity and additional impairment. The psychiatric comorbidity (sharing 
of symptoms across disorders) is high in adults with NPD and, in spite of clinical practice becoming increasingly specialized in the past few decades (with healthcare services catered to either ASD or ADHD or other neurodevelopmental disorders such as Tourette syndrome only), there is a growing realization that coexistence of disorders constitutes the rule rather than the exception [61] [62].

\section{Acknowledgements}

The authors like to thank the coordinators and coaches who took part in the interviews. This work was supported by The Health and Medical Care Committee of the Regional Executive Board, Region Västra Götaland.

\section{Disclosures}

Other than the financial disclosures already made, the authors have nothing to declare.

\section{References}

[1] Renty, J.O. and Roeyers, H. (2006) Quality of Life in High-Functioning Adults with Autism Spectrum Disorder. The Predictive Value of Disability and Support Characteristics. Autism, 10, 511-524. https://doi.org/10.1177/1362361306066604

[2] Taylor, N., Fauset, A. and Harpin, V. (2010) Young Adults with ADHD: An Analysis of Their Service Needs on Transfer to Adult Services. Archives of Disease in Childhood, 95, 513-517. https://doi.org/10.1136/adc.2009.164384

[3] Kolar, D., Keller, A., Golfinopoulos, M., Cumyn, L., Syer, C. and Hechtman, L. (2008) Treatment of Adults with Attention-Deficit/Hyperactivity Disorder. Neuropsychiatric Disease and Treatment, 4, 389-403.

[4] Torgersen, T., Gjervan, B. and Rasmussen, K. (2008) Treatment of Adult ADHD: Is Current Knowledge Useful to Clinicians? Neuropsychiatric Disease and Treatment, 4, 177-186. https://doi.org/10.2147/NDT.S1223

[5] Levine, M. (2005) Ready or Not, Here Life Comes. Simon \& Schuster, New York.

[6] Bellini, S. (2004) Social Skill Deficits and Anxiety in High-Functioning Adolescents with Autism Spectrum Disorders. Focus on Autism and Other Developmental Disabilities, 19, 78-86. https://doi.org/10.1177/10883576040190020201

[7] Farrugia, S. and Hudson, J. (2006) Anxiety in Adolescents with Asperger Syndrome: Negative Thoughts, Behavioral Problems, and Life Interference. Focus on Autism and Other Developmental Disabilities, 21, 25-35.

https://doi.org/10.1177/10883576060210010401

[8] Singh, S.P., Paul, M., Ford, T., Kramer, T., Weaver, T., McLaren, S., Hovish, K., Islam, Z., Belling, R. and White, S. (2010) Process, Outcome and Experience of Transition from Child to Adult Mental Healthcare: Multiperspective Study. The British Journal of Psychiatry, 197, 305-312. https://doi.org/10.1192/bjp.bp.109.075135

[9] Nadig, A., Lee, I., Singh, L., Bosshart, K. and Ozonoff, S. (2010) How Does the Topic of Conversation Affect Verbal Exchange and Eye Gaze? A Comparison between Typical Development and High-Functioning Autism. Neuropsychologia, 48, 2730 2739. https://doi.org/10.1016/j.neuropsychologia.2010.05.020

[10] Wentz, E., Nydén, A. and Krevers, B. (2012) Development of an Internet-Based Support and Coaching Model for Adolescents and Young Adults with ADHD and Autism Spectrum Disorders: A Pilot Study. European Child \& Adolescent Psychia- 
try, 21, 611-622. https://doi.org/10.1007/s00787-012-0297-2

[11] Jordan, C.J. (2010) Evolution of Autism Support and Understanding via the World Wide Web. Intellectual and Developmental Disabilities, 48, 220-227. https://doi.org/10.1352/1934-9556-48.3.220

[12] Sleeper-Triplett, J. (2008) The Effectiveness of Coaching for Children and Teens with AD/HD. Pediatric Nursing, 34, 433-435.

[13] Mesibov, G.B. (1984) Social Skills Training with Verbal Autistic Adolescents and Adults: A Program Model. Journal of Autism and Developmental Disorders, 14, 395-404. https://doi.org/10.1007/BF02409830

[14] Bodenheimer, T. (1999) The American Health Care System: The Movement for Improved Quality in Health Care. The New England Journal of Medicine, 340, 488-492. https://doi.org/10.1056/NEJM199902113400621

[15] Bero, L., Grilli, R., Grimshaw, J., Harvey, E., Oxman, A.D. and Thomson, M.A. (1998) Closing the Gap between Research and Practice: An Overview of Systematic Reviews of Interventions to Promote the Implementation of Research Findings. BMJ, 317, 465-468. https://doi.org/10.1136/bmj.317.7156.465

[16] Watson, R., Parr, J.R., Joyce, C., May, C. and Le Couteur, A.S. (2011) Models of Transitional Care for Young People with Complex Health Needs: A Scoping Review. Child: Care, Health and Development, 37, 780-791. https://doi.org/10.1111/j.1365-2214.2011.01293.x

[17] Acocella, I. (2012) Thefocus Groups in Social Research: Advantages and Disadvantages. Quality \& Quantity, 46, 1125-1136. https://doi.org/10.1007/s11135-011-9600-4

[18] Britten, N. (1999) Qualitative Interviews in Healthcare. In: Pope, C. and Mays, N., Eds., Qualitative Research in Health Care, 2nd Edition, BMJ Books, London, 11-19.

[19] Braun, V. and Clarke, V. (2006) Using Thematic Analysis in Psychology. Qualitative Research in Psychology, 3, 77-101. https://doi.org/10.1191/1478088706qp063oa

[20] Fixsen, D.L., Naoom, S.F., Blase, K.A., Friedman, R.M. and Wallace, F. (2005) Implementation Research: A Synthesis of the Literature. University of South Florida, Louise de la Parte Florida Mental Health Institute, the National Implementation Research Network, Tampa.

[21] Greenhalgh, T., Robert, G., Bate, P., Macfarlane, F. and Kyriakidou, O. (2005) Diffusion of Innovations in Health Service Organizations: A Systematic Literature Review. Blackwell Publishing, Oxford. https://doi.org/10.1002/9780470987407

[22] Denis, J.L., Hébert, Y., Langley, A., Lozeau, D. and Trottier, L.H. (2002) Explaining Diffusion Patterns for Complex Health Care Innovations. Health Care Manage Review, 27, 60-73. https://doi.org/10.1097/00004010-200207000-00007

[23] Craig, J. and Patterson, V. (2005) Introduction to the Practice of Telemedicine. Journal of Telemedicine and Telecare, 11, 3-9. https://doi.org/10.1258/1357633053430494

[24] Sood, S., Mbarika, V., Jugoo, S., Dookhy, R., Doarn, C.R., Prakash, N. and Merrell, R.C. (2007) What Is Telemedicine? A Collection of 104 Peer-Reviewed Perspectives and Theoretical Underpinnings. Telemedicine and E-Health, 13, 573-590. https://doi.org/10.1089/tmj.2006.0073

[25] Lehoux, P., Sicotte, C., Denis, J.L., Berg, M. and Lacroix, A. (2002) The Theory of Use behind Telemedicine: How Compatible with Physicians' Clinical Routine? Social Science \& Medicine, 54, 889-904. https://doi.org/10.1016/S0277-9536(01)00063-6

[26] Shiferaw, F. and Zolfo, M. (2012) The Role of Information Communication Tech- 
nology (ICT) towards Universal Health Coverage: The First Steps of a Telemedicine Project in Ethiopia. Global Health Action, 5, 15638. https://doi.org/10.3402/gha.v5i0.15638

[27] Oh, H., Rizo, C., Enkin, M. and Jadad, A. (2005) What Is eHealth (3): A Systematic Review of Published Definitions. Journal of Medical Internet Research, 7, e1. https://doi.org/10.2196/jmir.7.1.e1

[28] Eysenbach, G. (2001) What Is E-Health? Journal of Medical Internet Research, 3, e20. https://doi.org/10.2196/jmir.3.2.e20

[29] Heinzelmann, P.J., Lugn, N.E. and Kvedar, J.C. (2005) Telemedicine in the Future. Journal of Telemedicine and Telecare, 11, 384-390. https://doi.org/10.1258/135763305775013554

[30] Jennett, P.A., Affleck Hall, L., Hailey, D., Ohinmaa, A., Anderson, C., Thomas, R., Young, B., Lorenzetti, D. and Scott, R.E. (2003) The Socio-Economic Impact of Telehealth: A Systematic Review. Journal of Telemedicine and Telecare, 9, 311-320. https://doi.org/10.1258/135763303771005207

[31] Lehoux, P., Battista R.N. and Lance, J.-M. (2000) Telehealth: Passing Fad or Lasting Benefits? Canadian Journal of Public Health, 91, 277-280.

[32] Blaya, J.A., Fraser, H.S.F. and Holt, B. (2010) E-Health Technologies Show Promise Health Affairs, 29, 244-251. https://doi.org/10.1377/hlthaff.2009.0894

[33] Marcin, J.P., Ellis, J., Mawis, R., Nagrampa, E., Nesbitt, T.S. and Dimand, R.J. (2004) Using Telemedicine to Provide Pediatric Subspecialty Care to Children with Special Health Care Needs in an Underserved Rural Community. Pediatrics, 113, 1-6. https://doi.org/10.1542/peds.113.1.1

[34] Mishra, S.K., Kapoor, L. and Singh, I.P. (2009) Telemedicine in India: Current Scenario and the Future. Telemedicine and E-Health, 15, 568-575.

https://doi.org/10.1089/tmj.2009.0059

[35] Mair, F.S., May, C., Finch, T., Murray, E., Anderson, G., Sullivan, F., O’Donnell, C., Wallace, P. and Epstein, O. (2007) Understanding the Implementation and Integration of E-Health Services. Journal of Telemedicine and Telecare, 13, 36-37. https://doi.org/10.1258/135763307781645112

[36] Chiasson, M.W. and Davidson, E. (2004) Pushing the Contextual Envelope: Developing and Diffusing IS Theory for Health Information Systems Research. Information and Organization, 14, 155-188. https://doi.org/10.1016/j.infoandorg.2004.02.001

[37] Aas, M.I. (2001) A Qualitative Study of the Organizational Consequences of Telemedicine. Journal of Telemedicine and Telecare, 7, 18-26. https://doi.org/10.1258/1357633011936093

[38] May, C., Gask, L., Atkinson, T., Ellis, N., Mair, F. and Esmail, A. (2001) Resisting and Promoting New Technologies in Clinical Practice: The Case of Telepsychiatry. Social Science \& Medicine, 52, 1889-1901. https://doi.org/10.1016/S0277-9536(00)00305-1

[39] Pinto, J.K. and Slevin, D.P. (1988) Critical Success Factors in Effective Project Implementation. In: Cleland, D.I. and King, W.R., Eds., Project Management Handbook, 2nd Edition, Van Nostrand Reinhold, New York, 902-909.

[40] Klein, K.J. and Sorra, J.S. (1996) The Challenge of Innovation Implementation. Academy of Management Review, 21, 1055-1080.

[41] Rogers, E.M. (2003) Diffusion of Innovations. 5th Edition, Free Press, London.

[42] Aubert, B.A. and Hamel, G. (2001) Adoption of Smart Cards in the Medical Sector: The Canadian Experience. Social Science \& Medicine, 53, 879-894. 
https://doi.org/10.1016/S0277-9536(00)00388-9

[43] Rohrbach, L.A., Grana, R., Sussman, S. and Valente, T.W. (2006) Type II Translation: Transporting Prevention Interventions from Research to Real-World Settings. Evaluation \& the Health Professions, 29, 302-333. https://doi.org/10.1177/0163278706290408

[44] Meyer, A. and Goes, J. (1988) Organizational Assimilation of Innovations: A Multilevel Contextual Analysis. Academy of Management Journal, 31, 897-923. https://doi.org/10.2307/256344

[45] Glaser, E.M. and Backer, T.E. (1980) Durability of Innovations: How Goal Attainment Scaling Programs Fare over Time. Community Mental Health Journal, 16, 130-143. https://doi.org/10.1007/BF00778585

[46] McLaren, P. (2003) Telemedicine and Telecare: What Can It offer Mental Health Services? Advances in Psychiatric Treatment, 9, 54-61. https://doi.org/10.1192/apt.9.1.54

[47] Tangalos, E.G. (1995) Enabling Technologies for Telepresence. Proceedings of the National Forum: Military Telemedicine On-Line Today: Research, Practice, and Opportunities, McLean, March 27-29, 5-20.

[48] Aranda, K. (2005) Community Nurses' Talk of Equality and the Discursive Constitution of Selves. Journal of Advanced Nursing, 51, 131-139. https://doi.org/10.1111/j.1365-2648.2005.03476.x

[49] Haug, M. R. (1988) Power, Authority and Health Behavior. In: Gochman, D.S., Ed., Health Behavior. Emerging Research Perspectives, Plenum Press, New York, 325336. https://doi.org/10.1007/978-1-4899-0833-9_18

[50] Little, P., Everitt, H., Williamson, I., Warner, G., Moore, M., Gould, C., Ferrier, K. and Payne, S. (2001) Preference of Patients for Patient Centered Approach to Consultation in Primary Care: Observational Study. BMJ, 322, 468-472.

https://doi.org/10.1136/bmj.322.7284.468

[51] Sackett, D.L., Rosenberg, W.M.C., Gray, J.A., Haynes, R.B. and Richardson, W.S. (1996) Evidence-Based Medicine: What It Is, and What Isn't It. BMJ, 312, 71-72. https://doi.org/10.1136/bmj.312.7023.71

[52] Laine, C. and Davidoff, F. (1996) Patient-Centered Medicine. A Professional Evolution. JAMA, 275, 152-156. https://doi.org/10.1001/jama.1996.03530260066035

[53] Gerteis, M., Edgman-Levitan, S., Daley, J. and Delbanco, T.L. (1993) Through the Patient's Eyes: Understanding and Promoting Patient-Centered Care. Jossey-Bass, San Francisco.

[54] Mair, F.S., May, C., O’Donnell, C., Finch, T., Sullivan, F. and Murray, E. (2012) Factors That Promote or Inhibit the Implementation of E-Health Systems: An Explanatory Systematic Review. Bulletin of the World Health Organization, 90, $357-$ 364. https://doi.org/10.2471/BLT.11.099424

[55] Helfrich, C.D., Weiner, B.J., McKinney, M.M. and Minasian, L. (2007) Determinants of Implementation Effectiveness. Adapting a Framework for Complex Innovations. Medical Care Research and Review, 64, 279-303. https://doi.org/10.1177/1077558707299887

[56] Ashforth, B. and Mael, F.A. (1998) The Power of Resistance: Sustaining Valued Identities. In: Kramer, R.M. and Neale, M.A., Eds., Power and Influence in Organizations, Sage, Thousand Oaks, 89-120. https://doi.org/10.4135/9781483345291.n5

[57] Brower, R.S. and Abolafia, M.F. (1995) The Structural Embeddedness of Resistance among Public Managers. Group Organization Management, 20, 149-166. https://doi.org/10.1177/1059601195202005

[58] Ford, E.W., Menachemi, N., Peterson, L.T. and Huerta, T.R. (2009) Resistance Is 
Futile: But It Is Slowing the Pace of EHR Adoption Nonetheless. Journal of the American Medical Informatics Association, 16, 274-281. https://doi.org/10.1197/jamia.M3042

[59] Lagarde, M., Smith Paintain, L., Antwi, G., Jones, C., Greenwood, B., Chandramohan, D., Tagbor, H. and Webster, J. (2011) Evaluating Health Workers' Potential Resistance to New Interventions: A Role for Discrete Choice Experiments. PLoS ONE, 6, e23588. https://doi.org/10.1371/journal.pone.0023588

[60] Jones, R.A., Jimmieson, N.L. and Griffiths, A. (2005) The Impact of Organizational Culture and Reshaping Capabilities on Change Implementation Success: The Mediating Role of Readiness for Change. Journal of Management Studies, 42, 361-386. https://doi.org/10.1111/j.1467-6486.2005.00500.x

[61] Gillberg, C. (2010) The ESSENCE in Child Psychiatry: Early Symptomatic Syndromes Eliciting Neurodevelopmental Clinical Examinations. Research in Developmental Disabilities, 31, 1543-1551. https://doi.org/10.1016/j.ridd.2010.06.002

[62] Kadesjö, B. and Gillberg, C. (2001) The Comorbidity of ADHD in the General Population of Swedish School Age Children. Journal of Child Psychology and Psychiatry, 42, 487-492. https://doi.org/10.1111/1469-7610.00742

[63] Ferlie, E.B. and Shortell, S.M. (2001) Improving the Quality of Health Care in the United Kingdom and the United States: A Framework for Change. Milbank Quarterly, 79, 281-315. https://doi.org/10.1111/1468-0009.00206

[64] Glisson, C. and Schoenwald, S. (2005) The ARC Organizational and Community Intervention Strategy for Implementing Evidence-Based Children's Mental Health Treatments. Mental Health Services Research, 7, 243-259.

https://doi.org/10.1007/s11020-005-7456-1

[65] Grimshaw, J., Shirran, L., Thomas, R., Mowatt, G., Fraser, C., Bero, L., Grilli, R, Harvey, E., Oxman, A. and O’Brien, M.A. (2001) Changing Provider Behaviour: An Overview of Systematic Reviews of Interventions. Medical Care, 39, II2-II45. https://doi.org/10.1097/00005650-200108002-00002

[66] Grol, R. and Grimshaw, J. (1999) Evidence-Based Implementation of EvidenceBased Medicine. The Joint Commission Journal on Quality Improvement, 25, 503 513. https://doi.org/10.1016/S1070-3241(16)30464-3

[67] Grol, R. and Grimshaw, J. (2003) From Best Evidence to Best Practice: Effective Implementation of Change in Patients' Care. Lancet, 362, 1225-1230. https://doi.org/10.1016/S0140-6736(03)14546-1

[68] HedmanAhlström, B. and Wentz, E. (2015) Performance and Social Life Perceived by Young Persons with ADHD and Autism: A Chat-Log Analysis. Psychology Research, 5, 114-124.

[69] HedmanAhlström, B. and Wentz, E. (2014) Difficulties in Everyday Life: Young Persons with Attention-Deficit/Hyperactivity Disorder and Autism Spectrum Disorders Perspectives. A Chat-Log Analysis. International Journal of Qualitative Studies on Health and Well-Being, 9, 23376.

[70] Hoagwood, K., Hibbs, E., Brent, D. and Jensen, P.J. (1995) Efficacy and Effectiveness in Studies of Child and Adolescent Psychotherapy. Journalof Consulting and Clinical Psychology, 63, 683-687. https://doi.org/10.1037/0022-006X.63.5.683

[71] Guldbrandsson, E. (2007) Från nyhet till vardagsnytta: Om implementeringens mödosamma konst: En Forskningssammanställning. Statens Folkhälsoinstitut, 20.

[72] Kazdin, A.E. (2008) Evidence-Based Treatment and Practice. New Opportunities to Bridge Clinical Research and Practice, Enhance the Knowledge Base, and Improve Patient Care. American Psychologist, 63, 146-159.

https://doi.org/10.1037/0003-066X.63.3.146 
Submit or recommend next manuscript to SCIRP and we will provide best service for you:

Accepting pre-submission inquiries through Email, Facebook, LinkedIn, Twitter, etc. A wide selection of journals (inclusive of 9 subjects, more than 200 journals)

Providing 24-hour high-quality service

User-friendly online submission system

Fair and swift peer-review system

Efficient typesetting and proofreading procedure

Display of the result of downloads and visits, as well as the number of cited articles Maximum dissemination of your research work

Submit your manuscript at: http://papersubmission.scirp.org/

Or contact health@scirp.org 